PROCEEDINGS OF THE

AMERICAN MATHEMATICAL SOCIETY

Volume 138, Number 1, January 2010, Pages 85-99

S 0002-9939(09)10039-4

Article electronically published on August 25, 2009

\title{
AN ANALOG OF KOSTANT'S THEOREM FOR THE COHOMOLOGY OF QUANTUM GROUPS
}

\author{
UNIVERSITY OF GEORGIA VIGRE ALGEBRA GROUP
}

(Communicated by Gail R. Letzter)

\begin{abstract}
We prove the analog of Kostant's Theorem on Lie algebra cohomology in the context of quantum groups. In particular, it is shown that Kostant's cohomology formula holds for quantum groups at a generic parameter $q$, recovering an earlier result of Malikov in the case where the underlying semisimple Lie algebra $\mathfrak{g}=\mathfrak{s l}(n)$. We also show that Kostant's formula holds when $q$ is specialized to an $\ell$-th root of unity for odd $\ell \geq h-1$ (where $h$ is the Coxeter number of $\mathfrak{g}$ ) when the highest weight of the coefficient module lies in the lowest alcove. This can be regarded as an analog of results of Friedlander-Parshall and Polo-Tilouine on the cohomology of Lie algebras of reductive algebraic groups in prime characteristic.
\end{abstract}

\section{INTRODUCTION}

1.1. Let $\mathfrak{g}$ be a complex semisimple Lie algebra, $L(\lambda)$ be a finite dimensional $\mathfrak{g}$ module, $\mathfrak{p}_{J}$ be a parabolic subalgebra, $\mathfrak{u}_{J}$ be the nilradical of $\mathfrak{p}_{J}$ and $\mathfrak{l}_{J}$ be the associated Levi subalgebra. A celebrated result of Kostant states that the cohomology $\mathrm{H}^{\bullet}\left(\mathfrak{u}_{J}, L(\lambda)\right)$ is the (multiplicity-free) direct sum of finite dimensional $\mathfrak{l}_{J}$-modules, $L_{J}(w \cdot \lambda)$, with $w \in{ }^{J} W$ (minimal length coset representatives of $W_{J} \backslash W$ where $W_{J}$ is the Weyl group associated to the subset $J$ of simple roots). There are many proofs of this result (e.g. [Kna, GW]), including ones which involve constructing BGG resolutions $[\mathrm{RC}, \mathrm{HeK}$. Recently the authors $\mathrm{UGA}$ provided a proof of Kostant's theorem by utilizing linkage in the parabolic category $\mathcal{O}_{J}$.

In this paper we will prove an analog of Kostant's theorem in the case of quantum groups over generic parameters and at roots of unity. Our proof uses many of the ideas presented in [UGA. More specifically, we first consider a subalgebra $\mathcal{U}_{q}\left(\mathfrak{u}_{J}\right)$ of the quantized enveloping algebra $\mathcal{U}_{q}(\mathfrak{g})$ which is explicitly defined in $\mathrm{BNPP}, \S 2.4$, $\S 2.6]$ using deep results of Lusztig on the existence of a PBW basis. In the classical case, the universal enveloping algebra of $\mathfrak{u}_{J}$ is a Hopf algebra and the cohomology of this algebra can be computed by using a complex which determines the (ordinary) Lie algebra cohomology. However, in the quantum case the subalgebra $\mathcal{U}_{q}\left(\mathfrak{u}_{J}\right)$ is an augmented algebra but not a Hopf algebra. Moreover, the cohomology for $\mathcal{U}_{q}\left(\mathfrak{u}_{J}\right)$ is

Received by the editors September 8, 2008, and, in revised form, September 28, 2008, and May 14, 2009.

2000 Mathematics Subject Classification. Primary 20G42.

The members of the UGA VIGRE Algebra Group are Irfan Bagci, Brian D. Boe, Leonard Chastkofsky, Benjamin Connell, Benjamin Jones, Wenjing Li, Daniel K. Nakano, Kenyon J. Platt, Jae-Ho Shin, and Caroline B. Wright.

(C)2009 American Mathematical Society Reverts to public domain 28 years from publication 
much more difficult to compute than in the classical case because of the lack of an explicit complex. This necessitates the use of other techniques involving spectral sequences and Euler characteristics.

We consider finite dimensional modules $L^{q}(\lambda)$ for $\mathcal{U}_{q}(\mathfrak{g})$, and calculate the cohomology

$$
\mathrm{H}^{\bullet}\left(\mathcal{U}_{q}\left(\mathfrak{u}_{J}\right), L^{q}(\lambda)\right):=\operatorname{Ext}_{\mathfrak{U}_{q}\left(\mathfrak{u}_{J}\right)}\left(\mathbb{C}, L^{q}(\lambda)\right)
$$

as a $\mathcal{U}_{q}\left(\mathfrak{l}_{J}\right)$-module. In the case when $q$ is a generic parameter our results recover an earlier result of Malikov Mal] when $\mathfrak{g}=\mathfrak{s l}(n)$ and $\mathfrak{u}_{J}=\mathfrak{u}$ is the unipotent radical of a Borel subalgebra. We also consider the case when $q$ is specialized to an $l$ th root of unity. In this situation, when $l \geq h-1$ ( $h$ being the Coxeter number of the underlying root system), we prove a version of Kostant's theorem for weights in the lowest alcove. These results can be regarded as an analog for quantum groups of results by Friedlander and Parshall [FP] and by Polo and Tilouine [PT, Corollary 2.10 ] for the cohomology of Lie algebras of reductive algebraic groups in prime characteristic.

1.2. The paper is organized as follows. The basic information we shall need about quantum groups is in Section 2. In Section 3 we introduce a parabolic version of Category $\mathcal{O}$ for quantum groups, which generalizes the ordinary quantum group Category $\mathcal{O}^{q}$ (as defined in $[\mathrm{HK}]$ ). Within these categories blocks can be defined and the simple modules in a block are parametrized by standard "linkage classes". This information gives us upper bounds on the composition factors in the cohomology. In Section 4, we provide results which compare the Euler characteristics on the ordinary $\mathfrak{u}_{J}$-cohomology with the cohomology for $\mathcal{U}_{q}\left(\mathfrak{u}_{J}\right)$. These techniques provide effective lower bounds on the composition factors in $\mathrm{H}^{\bullet}\left(\mathcal{U}_{q}\left(\mathfrak{u}_{J}\right), L^{q}(\lambda)\right)$. With the results in Sections 3 and 4 we prove the quantum version of Kostant's theorem in Section 5 (Theorems 5.1.1 and 5.2.1). In Section 6 we apply linkage in a graded version of the small quantum group $u_{\zeta}(\mathfrak{g})$, where $\zeta$ is a primitive $l$ th root of unity, along with the Euler characteristic results in Section 4 to prove the quantum version of the Polo-Tilouine result.

\section{Quantum GRoups}

2.1. We will follow the conventions as described in BNPP, Section 2]. Let $\mathfrak{g}$ be a complex simple Lie algebra. Let $\Phi$ be the irreducible root system associated to $\mathfrak{g}$ and $\Delta=\left\{\alpha_{1}, \ldots, \alpha_{r}\right\}$ be a fixed set of simple roots. The set $\Phi$ spans a real vector space $\mathbb{E}$ with positive definite inner product $\langle u, v\rangle, u, v \in \mathbb{E}$, normalized so that $\langle\alpha, \alpha\rangle=2$ if $\alpha \in \Phi$ is a short root. For $\alpha \in \Phi$, let $\alpha^{\vee}=\frac{2}{\langle\alpha, \alpha\rangle} \alpha$. For $J \subseteq \Delta$, let $\Phi_{J}=\Phi \cap \mathbb{Z} J$ be the root subsystem of $\Phi$ generated by $J$. Let $W$ be the Weyl group corresponding to $\Phi$ and $W_{J}$ the Weyl group of $\Phi_{J}$, viewed as a subgroup of $W$. Let ${ }^{J} W$ denote the set of minimal length coset representatives for $W_{J} \backslash W$.

Define the fundamental dominant weights $\varpi_{1}, \cdots, \varpi_{r}$ by $\left\langle\varpi_{i}, \alpha_{j}^{\vee}\right\rangle=\delta_{i, j}$, so that the (integral) weight lattice is $X=\mathbb{Z} \varpi_{1} \oplus \cdots \oplus \mathbb{Z} \varpi_{r}$ and the set of dominant (integral) weights is $X^{+}=\mathbb{N} \varpi_{1} \oplus \cdots \oplus \mathbb{N} \varpi_{r}$, where $\mathbb{N}$ denotes the set of nonnegative integers. Let

$$
X_{J}^{+}=\{\lambda \in X:\langle\lambda, \check{\alpha}\rangle \in \mathbb{N} \text { for all } \alpha \in J\}
$$

be the set of $J$-dominant integral weights. Set $\rho=\varpi_{1}+\cdots+\varpi_{r}$. For $\lambda \in X$ and $w \in W$ define the usual dot action $w \cdot \lambda=w(\lambda+\rho)-\rho$. 
Let $\mathfrak{t}$ be a fixed maximal toral subalgebra of $\mathfrak{g}$. Given $\alpha \in \Phi$, let $\mathfrak{g}_{\alpha}$ be the $\alpha$-root space. Put $\mathfrak{b}^{+}=\mathfrak{t} \oplus \bigoplus_{\alpha \in \Phi^{+}} \mathfrak{g}_{\alpha}$ (the positive Borel subalgebra) and $\mathfrak{b}=$ $\mathfrak{t} \oplus \bigoplus_{\alpha \in \Phi^{-}} \mathfrak{g}_{\alpha}$ (the opposite Borel subalgebra). More generally, given a subset $J \subseteq \Delta$, one can consider the Levi and parabolic Lie subalgebras $\mathfrak{l}_{J}$ and $\mathfrak{p}_{J}=\mathfrak{l}_{J} \oplus \mathfrak{u}_{J}$ of $\mathfrak{g}$. Here $\mathfrak{l}_{J}$ is the sum of $\mathfrak{t}$ and the root spaces $\mathfrak{g}_{\alpha}$ for which $\alpha$ is in the span of $J$, and $\mathfrak{u}_{J}$ is the sum of the root spaces $\mathfrak{g}_{-\alpha}$ for $\alpha \in \Phi^{+} \backslash \Phi_{J}^{+}$. When $J=\varnothing$ we have $\mathfrak{l}_{J}=\mathfrak{t}$ and $\mathfrak{p}_{J}=\mathfrak{b}$.

2.2. Throughout this paper let $l>1$ be a fixed odd positive integer. If $\Phi$ has type $G_{2}$, then we assume that 3 does not divide $l$. Let $\mathcal{A}=\mathbb{Q}\left[q, q^{-1}\right]$ with fraction field $\mathbb{Q}(q)$. Let $\zeta=\sqrt[l]{1} \in \mathbb{C}$ be a primitive $l$ th root of unity and $k=\mathbb{Q}(\zeta)$. One can regard $k$ as an $\mathcal{A}$-algebra by means of the homomorphism $\mathbb{Q}\left[q, q^{-1}\right] \rightarrow k$ where $q \mapsto \zeta$.

The quantized enveloping algebra $\mathbb{U}_{q}(\mathfrak{g})$ of $\mathfrak{g}$ is the $\mathbb{Q}(q)$-algebra with generators $E_{\alpha}, K_{\alpha}^{ \pm 1}, F_{\alpha}, \alpha \in \Delta$, and relations (R1)-(R6) listed in [Jan3, (4.3)]. The algebra $\mathbb{U}_{q}(\mathfrak{g})$ has two $\mathcal{A}$-forms, $U_{q}^{\mathcal{A}}(\mathfrak{g})$ (due to Lusztig) and $\mathcal{U}_{q}^{\mathcal{A}}(\mathfrak{g})$ (due to De Concini and $\mathrm{Kac})$. Often we will use $\mathcal{U}_{q}(\mathfrak{g})$ to denote the latter $\mathcal{A}$-form specialized to a generic parameter. After a base change to $k$, these algebras play roles analogous to the hyperalgebra of a reductive group and the universal enveloping algebra of its Lie algebra, respectively. Set

$$
U_{\zeta}(\mathfrak{g}):=k \otimes_{\mathcal{A}} U_{q}^{\mathcal{A}}(\mathfrak{g}) /\left\langle 1 \otimes K_{\alpha}^{l}-1 \otimes 1, \alpha \in \Delta\right\rangle .
$$

The elements $E_{\alpha}, K_{\alpha}, F_{\alpha}, \alpha \in \Delta$, in $U_{\zeta}(\mathfrak{g})$ generate a Hopf subalgebra, denoted by $u_{\zeta}(\mathfrak{g})$, of $U_{\zeta}(\mathfrak{g})$.

Recall that $\mathcal{U}_{q}^{\mathcal{A}}(\mathfrak{g})$ is the $\mathcal{A}$-subalgebra of $\mathbb{U}_{q}(\mathfrak{g})$ generated by the $E_{\alpha}, F_{\alpha}, K_{\alpha}^{ \pm 1}$, $\alpha \in \Delta$. There is an inclusion of $\mathcal{A}$-forms: $\mathcal{U}_{q}^{\mathcal{A}}(\mathfrak{g}) \subseteq U_{q}^{\mathcal{A}}(\mathfrak{g})$. Now set $\mathcal{U}_{k}(\mathfrak{g}):=$ $k \otimes_{\mathcal{A}} \mathcal{U}_{q}^{\mathcal{A}}(\mathfrak{g})$. Finally, put

$$
\mathcal{U}_{\zeta}(\mathfrak{g})=\mathcal{U}_{k}(\mathfrak{g}) /\left\langle 1 \otimes K_{\alpha}^{l}-1 \otimes 1, \alpha \in \Delta\right\rangle .
$$

The (Hopf) algebra $\mathcal{U}_{\zeta}(\mathfrak{g})$ has a central subalgebra $\mathcal{Z}$ such that $u_{\zeta}(\mathfrak{g}) \cong \mathcal{U}_{\zeta}(\mathfrak{g}) / / \mathcal{Z}$ (cf. [DCK] for more details). The finite dimensional Hopf algebra $u_{\zeta}(\mathfrak{g})$ will be referred to as the small quantum group.

We will assume throughout that all $U_{q}(\mathfrak{g})$ and $U_{\zeta}(\mathfrak{g})$ modules we consider are integrable and of type 1 (cf. [BNPP, Section 2.2]). Given such a module $V$, let $w t(V)$ denote its set of weights.

2.3. Levi and parabolic subalgebras. The universal enveloping algebras of the Levi and parabolic subalgebras associated to $J$ will be denoted by $\mathbb{U}\left(\mathfrak{l}_{J}\right)$ and $\mathbb{U}\left(\mathfrak{p}_{J}\right)$. One can naturally define corresponding quantized enveloping algebras $\mathbb{U}_{q}\left(\mathfrak{l}_{J}\right)$ and $\mathbb{U}_{q}\left(\mathfrak{p}_{J}\right)$. As subalgebras of $\mathbb{U}_{q}(\mathfrak{g}), \mathbb{U}_{q}\left(\mathfrak{l}_{J}\right)$ is generated by $\left\{E_{\alpha}, F_{\alpha}: \alpha \in J\right\} \cup\left\{K_{\alpha}^{ \pm 1}\right.$ : $\alpha \in \Delta\}$, and $\mathbb{U}_{q}\left(\mathfrak{p}_{J}\right)$ is generated by $\left\{E_{\alpha}: \alpha \in J\right\} \cup\left\{F_{\alpha}, K_{\alpha}^{ \pm 1}: \alpha \in \Delta\right\}$. Upon specialization one obtains the subalgebras $U_{\zeta}\left(\mathfrak{l}_{J}\right), U_{\zeta}\left(\mathfrak{p}_{J}\right), u_{\zeta}\left(\mathfrak{l}_{J}\right), u_{\zeta}\left(\mathfrak{p}_{J}\right)$ of $U_{\zeta}(\mathfrak{g})$ and $\mathcal{U}_{\zeta}\left(\mathfrak{l}_{J}\right), \mathcal{U}_{\zeta}\left(\mathfrak{p}_{J}\right)$ of $\mathcal{U}_{\zeta}(\mathfrak{g})$. One can also make analogous constructions with the opposite parabolic $\mathfrak{p}_{J}^{+}$.

For each $\alpha \in \Delta$, Lusztig has defined an automorphism $T_{\alpha}$ of $\mathbb{U}_{q}(\mathfrak{g})$ (cf. [Jan3, Ch. 8]). If $s_{\alpha}$ is a simple reflection in $W$, let $T_{s_{\alpha}}:=T_{\alpha}$. More generally, given any $w \in W$, let $w=s_{\beta_{1}} s_{\beta_{2}} \cdots s_{\beta_{n}}$ be a reduced expression, and define $T_{w}:=$ $T_{\beta_{1}} \cdots T_{\beta_{n}} \in \operatorname{Aut}\left(\mathbb{U}_{q}(\mathfrak{g})\right)$. The automorphism $T_{w}$ is independent of the reduced expression of $w$. 
Now let $J \subseteq \Delta$ and fix a reduced expression for the longest element of $W$, $w_{0}=s_{\beta_{1}} \cdots s_{\beta_{N}}$, that starts with a reduced expression for the longest element $w_{0, J}$ of $W_{J}$. The fixed reduced expression for $w_{0}$ induces a fixed ordering on the positive roots, which in turn leads to a definition of "root vectors" $E_{\gamma}, F_{\gamma}$ for each $\gamma \in \Phi^{+}$ by using the automorphisms above, as in BNPP, Section 2.4]. Note that $E_{\gamma}$ has weight $\gamma$ and $F_{\gamma}$ has weight $-\gamma$. The vectors $E_{\gamma}, F_{\gamma}$, and $K_{\alpha}^{ \pm 1}$ for $\alpha \in \Delta$ form a PBW-like basis for $\mathbb{U}_{q}(\mathfrak{g})$. We also note that ordered monomials of elements $E_{\gamma}$, $F_{\gamma}$, where $\gamma \in \Phi^{+} \cap \Phi_{J}$, and $K_{\alpha}^{ \pm 1}$ for $\alpha \in \Delta$ form a PBW-like basis for $\mathbb{U}_{q}\left(\mathfrak{l}_{J}\right)$. Moreover, such a basis for $\mathbb{U}_{q}\left(\mathfrak{p}_{J}\right)$ can be obtained by taking ordered monomials in $E_{\gamma}, F_{\tau}$, where $\gamma \in \Phi^{+} \cap \Phi_{J}, \tau \in \Phi^{+}$, and $K_{\alpha}^{ \pm 1}$ for $\alpha \in \Delta$.

With the PBW basis as described above one can define a subalgebra $\mathbb{U}_{q}\left(\mathfrak{u}_{J}\right)$ which is analogous to that of $\mathbb{U}\left(\mathfrak{u}_{J}\right) \subset \mathbb{U}\left(\mathfrak{p}_{J}\right)$. Let $\Phi^{+} \backslash \Phi_{J}^{+}=\left\{\gamma_{i_{1}}, \ldots, \gamma_{i_{N}}\right\}$ and set $\mathbb{U}_{q}\left(\mathfrak{u}_{J}\right)$ to be the subspace spanned by the $F_{\gamma_{i_{1}}}^{a_{i_{1}}} \cdots F_{\gamma_{i_{N}}}^{a_{i_{N}}}, a_{i_{j}} \in \mathbb{Z}_{\geq 0}$. According to BNPP Lemma 2.4.1] $\mathbb{U}_{q}\left(\mathfrak{u}_{J}\right)$ is a subalgebra of $\mathbb{U}_{q}\left(\mathfrak{p}_{J}\right)$ and independent of the choice of reduced expression for $w_{0}$. Again by specializing one obtains algebras $U_{\zeta}\left(\mathfrak{u}_{J}\right)$ and $u_{\zeta}\left(\mathfrak{u}_{J}\right)$.

\section{Quantum PARABolic CATEgory $\mathcal{O}$}

3.1. Category $\mathcal{O}_{J}^{q}$. Define a partial order on $X$ by

$$
\mu \leq \lambda \Longleftrightarrow \lambda-\mu \text { is a sum of positive roots. }
$$

Given $\lambda \in X$, define

$$
D(\lambda)=\{\mu \in X: \mu \leq \lambda\} .
$$

The simple finite dimensional $\mathcal{U}_{q}\left(\mathfrak{l}_{J}\right)$-modules are parameterized by the set $X_{J}^{+}$. Denote the simple module of highest weight $\lambda \in X_{J}^{+}$by $L_{J}^{q}(\lambda)$.

Definition 3.1.1. Let $\mathcal{C}_{J}=\mathcal{C}\left(\mathcal{U}_{q}(\mathfrak{g}), \mathcal{U}_{q}\left(\mathfrak{l}_{J}\right)\right)$ denote the full subcategory of all $\mathcal{U}_{q}(\mathfrak{g})$-modules which are finitely semisimple as $\mathcal{U}_{q}\left(\mathfrak{l}_{J}\right)$-modules (that is, they decompose as a direct sum of finite dimensional simple $\mathcal{U}_{q}\left(\mathfrak{l}_{J}\right)$-modules where the simple modules occur with finite multiplicity).

Definition 3.1.2. Let $\mathcal{O}_{J}^{q}$ denote the full subcategory of $\mathcal{C}_{J}$ consisting of $\mathcal{U}_{q}(\mathfrak{g})$ modules $V$ which have finite dimensional weight spaces and satisfy the following condition: there exist $\lambda_{1}, \lambda_{2}, \ldots, \lambda_{t} \in X$ such that

$$
\operatorname{wt}(V) \subseteq D\left(\lambda_{1}\right) \cup D\left(\lambda_{2}\right) \cup \cdots \cup D\left(\lambda_{t}\right) .
$$

3.2. Verma modules. For each $\lambda \in X_{J}^{+}$, extend the finite dimensional irreducible $\mathcal{U}_{q}\left(\mathfrak{l}_{J}\right)$-module $L_{J}^{q}(\lambda)$ to a $\mathcal{U}_{q}\left(\mathfrak{p}_{J}\right)$-module by requiring that $F_{\gamma} L_{J}(\lambda)=0$ for all $\gamma \in \Phi^{+} \backslash \Phi_{J}^{+}$.

The parabolic Verma module corresponding to $\lambda \in X_{J}^{+}$is defined by

$$
Z_{J}^{q}(\lambda):=\mathcal{U}_{q}(\mathfrak{g}) \otimes_{\mathcal{U}_{q}\left(\mathfrak{p}_{J}\right)} L_{J}^{q}(\lambda)
$$

As in the classical setting (cf. [RC, Proposition 3.3]), the following properties hold:

(1) $Z_{J}^{q}(\lambda)$ is an object of $\mathcal{O}_{J}^{q}$.

(2) $Z_{J}^{q}(\lambda)$ is a highest weight module with highest weight $\lambda$.

(3) $Z_{J}^{q}(\lambda)$ has a unique maximal submodule and its unique irreducible quotient is isomorphic to $L^{q}(\lambda)$, the irreducible $\mathcal{U}_{q}(\mathfrak{g})$ module with highest weight $\lambda$.

(4) Every simple object in $\mathcal{O}_{J}^{q}$ is isomorphic to $L^{q}(\lambda)$ for some $\lambda \in X_{J}^{+}$. 
Specializing to $q=1$ (cf. [HK, Ch. 3]), we have an identification of the character of $L^{q}(\lambda)$ :

$$
\operatorname{ch} L^{q}(\lambda)=\operatorname{ch} L(\lambda)
$$

where $L(\lambda)$ denotes the irreducible $\mathbb{U}(\mathfrak{g})$ module with highest weight $\lambda$. (Note that this is not neccesarily finite dimensional since we only require that $\lambda \in X_{J}^{+}$.)

The Category $\mathcal{O}_{J}^{q}$ is a full subcategory of $\mathcal{O}^{q}$ (cf. [HK, $\left.\S 3.2\right]$ ). A crucial fact used in what follows is that the linkage principle holds in Category $\mathcal{O}_{J}^{q}$ since it holds in $\mathcal{O}^{q}$ (cf. Jan1, Claim 6.26]). In particular we use the following fact. Consider a weight $\nu \in X_{J}^{+}$. If $Z_{J}^{q}(\nu)$ has $L^{q}(\mu)$ as a composition factor, then $\nu=w \cdot \mu$ for some $w \in{ }^{J} W$.

3.3. Projective modules in $\mathcal{O}_{J}^{q}$. We have a decomposition of Category $\mathcal{O}_{J}^{q}$ into infinitesimal blocks

$$
\mathcal{O}_{J}^{q}=\bigoplus_{\mu \in \mathbf{t}^{*} / W} \mathcal{O}_{J}^{q}(\mu)
$$

In this section, let $Z(\lambda):=Z_{J}^{q}(\lambda)$ for brevity.

Proposition 3.3.1. The following hold:

(a) If $\lambda \in \mathfrak{t}^{*}$ is dominant, then $Z(\lambda)$ is projective.

(b) If $P \in \mathcal{O}_{J}^{q}$ is projective and $\operatorname{dim} L<\infty$, then $P \otimes L$ is projective in $\mathcal{O}_{J}^{q}$.

(c) Category $\mathcal{O}_{J}^{q}$ has enough projectives.

Proof. The proof follows the line of reasoning given for ordinary Category $\mathcal{O}$ in Hum. For completeness, we include the argument here.

(a) Start with an exact sequence $M \longrightarrow N \longrightarrow 0$ in $\mathcal{O}_{J}^{q}$. Suppose $\varphi: Z(\lambda) \longrightarrow N$ is a $\mathcal{U}_{q}(\mathfrak{g})$-module homomorphism. We want a map $\widetilde{\varphi}: Z(\lambda) \longrightarrow M$ such that

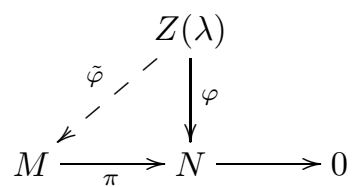

commutes. Since $Z(\lambda)$ is a highest weight module, it has a highest weight vector $v^{+}$, of weight $\lambda$. Consider $\varphi\left(v^{+}\right) \in N$. The map $\pi: M \longrightarrow N$ is surjective, so there exists $v \in M$ such that $\pi(v)=\varphi\left(v^{+}\right)$. Now, $Z(\lambda) \in \mathcal{O}_{J}^{q}(\lambda)$. Furthermore,

$$
N=\bigoplus_{\mu \in \mathfrak{t}^{*} / W} N^{\mu}
$$

with $N^{\mu} \in \mathcal{O}_{J}^{q}(\mu)$, and $\varphi(Z(\lambda)) \subseteq N^{\lambda}$. So without loss of generality, we may suppose $M, N \in \mathcal{O}_{J}^{q}(\lambda)$. The $\mathcal{U}_{q}\left(\mathfrak{n}^{+}\right)$-submodule of $M$ generated by $v$ has a maximal weight vector of weight $\mu \geq \lambda$. By the Linkage Principle, $\mu=w \cdot \lambda$ for some $w \in W$. Thus $\mu=\lambda$, since $\lambda$ is dominant. By the universal property of $Z(\lambda)$, there exists $\widetilde{\varphi}: Z(\lambda) \longrightarrow M$ such that $\widetilde{\varphi}\left(v^{+}\right)=v$ and (3.3.1) commutes. Hence $Z(\lambda)$ is projective.

(b) By the definition of Category $\mathcal{O}_{J}^{q}$, it is easy to see that $V \otimes L \in \mathcal{O}_{J}^{q}$ for any $V \in \mathcal{O}_{J}^{q}$ and any finite dimensional module $L$. In particular, $-\otimes L$ (and similarly $L \otimes-)$ are exact functors on $\mathcal{O}_{J}^{q}$. Since $P$ is projective if and only if $\operatorname{Hom}_{\mathcal{O}_{J}^{q}}(P,-)$ 
is an exact functor, $\operatorname{Hom}_{\mathcal{O}_{J}^{q}}(P, \operatorname{Hom}(L,-)) \cong \operatorname{Hom}_{\mathcal{O}_{J}^{q}}\left(P, L^{*} \otimes-\right)$ is the composition of two exact functors and hence exact. Furthermore,

$$
\operatorname{Hom}_{\mathcal{O}_{J}^{q}}(P \otimes L,-) \cong \operatorname{Hom}_{\mathcal{O}_{J}^{q}}(P, \operatorname{Hom}(L,-)) \text {. }
$$

Hence $\operatorname{Hom}_{\mathcal{O}_{J}^{q}}(P \otimes L,-)$ is exact, therefore $P \otimes L$ is projective in $\mathcal{O}_{J}^{q}$.

(c) We prove that if $M \in \mathcal{O}_{J}^{q}$, then there exists a projective module $P \in \mathcal{O}_{J}^{q}$ such that $P \rightarrow M$. We proceed by induction on the length of $M$. For the base step, for any $\lambda \in X_{J}^{+}$, we will find a projective $P \in \mathcal{O}_{J}^{q}$ such that $P \rightarrow L^{q}(\lambda)$. Let $\mu:=\lambda+n \rho$ for $n \geq 0$ large enough that $\mu$ is dominant; in particular, $\mu \in X_{J}^{+}$. Then the Verma module $Z(\mu)$ is projective by part (a). Since $n \rho \in X^{+}, L^{q}(n \rho)$ is finite dimensional. Hence $P:=Z(\mu) \otimes L^{q}(n \rho)$ is projective in $\mathcal{O}_{J}^{q}$ by part (b).

The standard argument using the tensor identity shows that if $\operatorname{dim} L<\infty$, then $Z(\xi) \otimes L$ has a finite Verma filtration with subquotients of the form $Z(\xi+\nu)$, where $\nu$ ranges over $\operatorname{wt}(L)$. Moreover, if $\nu$ is a minimal (respectively, maximal) weight of $L$, then $Z(\xi+\nu)$ is a quotient (resp., submodule) of $Z(\xi) \otimes L$. The lowest weight of $L^{q}(n \rho)$ is $-n \rho=w_{0}(n \rho)$, so $Z(\mu-n \rho)=Z(\lambda)$ is a quotient of $P$. Consequently,

$$
P \rightarrow Z(\lambda) \rightarrow L^{q}(\lambda)
$$

since $L^{q}(\lambda)$ is a quotient of $Z(\lambda)$.

For the inductive step, suppose $l(M)>1$ so that for some simple module $L^{q}(\lambda)$ $\left(\lambda \in X_{J}^{+}\right)$we have a short exact sequence

$$
0 \longrightarrow L(\lambda) \longrightarrow M \longrightarrow N \longrightarrow 0 .
$$

Now, $l(N)<l(M)$ and so by our induction hypothesis, there exists a projective module $Q \in \mathcal{O}_{J}^{q}$ such that we have a map $\varphi: Q \rightarrow N$. Consequently, there is a map $\widetilde{\varphi}: Q \rightarrow M$ such that

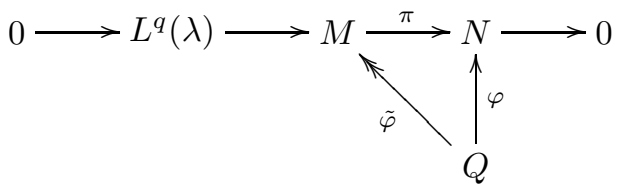

commutes. If $\widetilde{\varphi}$ is surjective, we are done. If $\widetilde{\varphi}$ is not surjective, then $M \cong$ $N \oplus L^{q}(\lambda)$. Consequently, taking $P \in \mathcal{O}_{J}^{q}$ such that $P$ is a projective cover of $L^{q}(\lambda)$, we have that $Q \oplus P \rightarrow M$.

3.4. Relative cohomology. In this subsection we outline basic definitions and results for relative cohomology. Let

$$
\cdots \rightarrow M_{i-1} \stackrel{f_{i-1}}{\longrightarrow} M_{i} \stackrel{f_{i}}{\rightarrow} M_{i+1} \rightarrow \cdots
$$

be a sequence of $\mathcal{U}_{q}(\mathfrak{g})$-modules. We say that this sequence is $\left(\mathcal{U}_{q}(\mathfrak{g}), \mathcal{U}_{q}\left(\mathfrak{l}_{J}\right)\right)$-exact if it is exact as a sequence of $\mathcal{U}_{q}(\mathfrak{g})$-modules and if, when viewed as a sequence of $\mathcal{U}_{q}\left(\mathfrak{l}_{J}\right)$-modules, $\operatorname{Ker} f_{i}$ is a direct summand of $M_{i}$ for all $i$.

A $\mathcal{U}_{q}(\mathfrak{g})$-module $P$ is $\left(\mathcal{U}_{q}(\mathfrak{g}), \mathcal{U}_{q}\left(\mathfrak{l}_{J}\right)\right)$-projective if given any $\left(\mathcal{U}_{q}(\mathfrak{g}), \mathcal{U}_{q}\left(\mathfrak{l}_{J}\right)\right)$-exact sequence

$$
0 \rightarrow M_{1} \stackrel{f}{\rightarrow} M_{2} \stackrel{g}{\rightarrow} M_{3} \rightarrow 0
$$

and $\mathcal{U}_{q}(\mathfrak{g})$-module homomorphism $h: P \rightarrow M_{3}$ there is a $\mathcal{U}_{q}(\mathfrak{g})$-homomorphism $\tilde{h}: P \rightarrow M_{2}$ satisfying $g \circ \tilde{h}=h$.

In particular, note that if $P$ is a projective $\mathcal{U}_{q}(\mathfrak{g})$-module, then it is automatically $\left(\mathcal{U}_{q}(\mathfrak{g}), \mathcal{U}_{q}\left(\mathfrak{l}_{J}\right)\right)$-projective. 
A $\left(\mathcal{U}_{q}(\mathfrak{g}), \mathcal{U}_{q}\left(\mathfrak{l}_{J}\right)\right)$-projective resolution of a $\mathcal{U}_{q}(\mathfrak{g})$-module is a $\left(\mathcal{U}_{q}(\mathfrak{g}), \mathcal{U}_{q}\left(\mathfrak{l}_{J}\right)\right)$ exact sequence

$$
\cdots \stackrel{\delta_{2}}{\rightarrow} P_{1} \stackrel{\delta_{1}}{\rightarrow} P_{0} \stackrel{\delta_{0}}{\rightarrow} M \rightarrow 0,
$$

where each $P_{i}$ is a $\left(\mathcal{U}_{q}(\mathfrak{g}), \mathcal{U}_{q}\left(\mathfrak{l}_{J}\right)\right)$-projective module. We record here a lemma which is a special case of a classical result due to Hochschild; see [Hoc, Lemma 2 and §2].

Lemma 3.4.1. The following statements hold:

(a) If $M$ is any $\mathcal{U}_{q}\left(\mathfrak{l}_{J}\right)$-module, then $\mathcal{U}_{q}(\mathfrak{g}) \otimes_{\mathcal{U}_{q}\left(\mathfrak{l}_{J}\right)} M$ is a $\left(\mathcal{U}_{q}(\mathfrak{g}), \mathcal{U}_{q}\left(\mathfrak{l}_{J}\right)\right)$-projective $\mathcal{U}_{q}(\mathfrak{g})$-module.

(b) Any $\mathcal{U}_{q}(\mathfrak{g})$-module admits a $\left(\mathcal{U}_{q}(\mathfrak{g}), \mathcal{U}_{q}\left(\mathfrak{l}_{J}\right)\right)$-projective resolution, namely,

$$
\ldots \stackrel{\delta_{2}}{\rightarrow} \mathcal{U}_{q}(\mathfrak{g}) \otimes_{\mathcal{U}_{q}\left(\mathfrak{l}_{J}\right)} \operatorname{Ker} \delta_{0} \stackrel{\delta_{7}}{\rightarrow} \mathcal{U}_{q}(\mathfrak{g}) \otimes_{\mathcal{U}_{q}\left(\mathfrak{l}_{J}\right)} M \stackrel{\delta_{0}}{\rightarrow} M \rightarrow 0 .
$$

Here $\delta_{i}$ is the "multiplication" map $\mathcal{U}_{q}(\mathfrak{g}) \otimes_{\mathcal{U}_{q}\left(\mathfrak{l}_{J}\right)} N \rightarrow N$ given by $x \otimes n \mapsto$ xn for any $\mathcal{U}_{q}(\mathfrak{g})$-module $N$.

Recall that the category $\mathcal{C}_{J}$ from Section 3 contains $\mathcal{O}_{J}^{q}$. A key connection between these categories is that if $M$ and $N$ are objects of $\mathcal{O}_{J}^{q}$, then one has

$$
\operatorname{Ext}_{\mathcal{O}_{J}^{q}}^{\bullet}(M, N) \cong \operatorname{Ext}_{\left(\mathcal{U}_{q}(\mathfrak{g}), \mathcal{U}_{q}\left(\mathfrak{l}_{J}\right)\right)}(M, N) \cong \operatorname{Ext}_{\mathcal{C}_{J}}^{\bullet}(M, N)
$$

(cf. $\mathrm{Kum}$ and $[\mathrm{BNW}$ ).

3.5. Composition factors. If $V$ is a finitely semisimple $\mathcal{U}_{q}\left(\mathfrak{l}_{J}\right)$-module, define $[V$ :

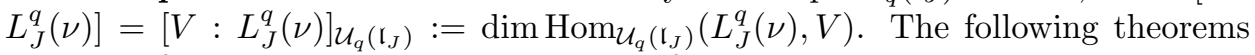
provide information about the composition factors inside the cohomology.

Theorem 3.5.1 (cf. [UGA, Theorem 2.3.1]). Let $V \in \mathcal{O}_{J}^{q}$ and let $\lambda \in X_{J}^{+}$.

(a) $\operatorname{Ext}_{\mathcal{O}_{J}^{q}}^{i}\left(Z_{J}^{q}(\lambda), V\right) \cong \operatorname{Hom}_{\mathcal{U}_{q}\left(\mathfrak{l}_{J}\right)}\left(L_{J}^{q}(\lambda), \mathrm{H}^{i}\left(\mathcal{U}_{q}\left(\mathfrak{u}_{J}\right), V\right)\right)$.

(b) If $\left[\mathrm{H}^{i}\left(\mathcal{U}_{q}\left(\mathfrak{u}_{J}\right), L^{q}(\mu)\right): L_{J}^{q}(\nu)\right] \neq 0$ for $\mu \in X^{+}$, then $\nu=w \cdot \mu$ for some $w \in{ }^{J} W$.

Proof. (a) First observe that $\operatorname{Ext}_{\mathcal{O}_{J}^{q}}^{i}\left(Z_{J}^{q}(\lambda), V\right) \cong \operatorname{Ext}_{\left(\mathcal{U}_{q}(\mathfrak{g}), \mathcal{U}_{q}\left(\mathfrak{I}_{J}\right)\right)}^{i}\left(Z_{J}^{q}(\lambda), V\right)$ and by Frobenius reciprocity we have

$$
\begin{aligned}
\operatorname{Ext}_{\left(\mathcal{U}_{q}(\mathfrak{g}), \mathcal{U}_{q}\left(\mathfrak{l}_{J}\right)\right)}^{i}\left(Z_{J}^{q}(\lambda), V\right) & \cong \operatorname{Ext}_{\left(\mathcal{U}_{q}\left(\mathfrak{p}_{J}\right), \mathcal{U}_{q}\left(\mathfrak{l}_{J}\right)\right)}\left(L_{J}^{q}(\lambda), V\right) \\
& \cong \mathrm{H}^{i}\left(\mathcal{U}_{q}\left(\mathfrak{p}_{J}\right), \mathcal{U}_{q}\left(\mathfrak{l}_{J}\right) ; L_{J}^{q}(\lambda)^{*} \otimes V\right) .
\end{aligned}
$$

One can use the Grothendieck spectral sequence construction given in Jan2, I, Proposition 4.1] to obtain a spectral sequence

$$
\begin{aligned}
E_{2}^{i, j} & =\mathrm{H}^{i}\left(\mathcal{U}_{q}\left(\mathfrak{p}_{J}\right) / / \mathcal{U}_{q}\left(\mathfrak{u}_{J}\right), \mathcal{U}_{q}\left(\mathfrak{l}_{J}\right) / \mathcal{U}_{q}\left(\mathfrak{l}_{J} \cap \mathfrak{u}_{J}\right) ; \mathrm{H}^{j}\left(\mathcal{U}_{q}\left(\mathfrak{u}_{J}\right), 0 ; L_{J}^{q}(\lambda)^{*} \otimes V\right)\right) \\
& \Rightarrow \mathrm{H}^{i+j}\left(\mathcal{U}_{q}\left(\mathfrak{p}_{J}\right), \mathcal{U}_{q}\left(\mathfrak{l}_{J}\right) ; L_{J}^{q}(\lambda)^{*} \otimes V\right)
\end{aligned}
$$

(see [GK, Sec. 5.2] for the definition of //). However,

$$
E_{2}^{i, j} \cong \mathrm{H}^{i}\left(\mathcal{U}_{q}\left(\mathfrak{l}_{J}\right), \mathcal{U}_{q}\left(\mathfrak{l}_{J}\right) ; \mathrm{H}^{j}\left(\mathcal{U}_{q}\left(\mathfrak{l}_{J}\right), 0 ; L_{J}^{q}(\lambda)^{*} \otimes V\right)\right)=0
$$

for $i>0$, so the spectral sequence collapses and yields

$$
\begin{aligned}
\operatorname{Hom}_{\mathcal{U}_{q}\left(\mathfrak{l}_{J}\right)}\left(L_{J}^{q}(\lambda), \mathrm{H}^{j}\left(\mathcal{U}_{q}\left(\mathfrak{u}_{J}\right), V\right)\right) & \cong \mathrm{H}^{0}\left(\mathcal{U}_{q}\left(\mathfrak{l}_{J}\right), \mathcal{U}_{q}\left(\mathfrak{l}_{J}\right) ; \mathrm{H}^{j}\left(\mathcal{U}_{q}\left(\mathfrak{u}_{J}\right), L_{J}^{q}(\lambda)^{*} \otimes V\right)\right) \\
& \cong \mathrm{H}^{j}\left(\mathcal{U}_{q}\left(\mathfrak{p}_{J}\right), \mathcal{U}_{q}\left(\mathfrak{l}_{J}\right) ; L_{J}^{q}(\lambda)^{*} \otimes V\right) .
\end{aligned}
$$


(b) Suppose that $\left[H^{i}\left(\mathcal{U}_{q}\left(\mathfrak{u}_{J}\right), L^{q}(\mu)\right): L_{J}^{q}(\nu)\right] \neq 0$. Then from part (a),

$$
\begin{aligned}
{\left[\mathrm{H}^{i}\left(\mathcal{U}_{q}\left(\mathfrak{u}_{J}\right), L^{q}(\mu)\right): L_{J}^{q}(\nu)\right]_{\mathcal{U}_{q}\left(\mathfrak{l}_{J}\right)} } & =\operatorname{dim} \operatorname{Hom}_{\mathcal{U}_{q}\left(\mathfrak{l}_{J}\right)}\left(L_{J}^{q}(\nu), H^{i}\left(\mathcal{U}_{q}\left(\mathfrak{u}_{J}\right), L^{q}(\mu)\right)\right) \\
& =\operatorname{dim}_{\operatorname{Ext}_{\mathcal{O}_{J}^{q}}^{i}\left(Z_{J}^{q}(\nu), L^{q}(\mu)\right)}
\end{aligned}
$$

But, $\operatorname{Ext}_{\mathcal{O}_{J}^{q}}^{i}\left(Z_{J}^{q}(\nu), L^{q}(\mu)\right) \neq 0$ implies by linkage that $\nu=w \cdot \mu$ for some $w \in{ }^{J} W$.

Theorem 3.5.2 (cf. [UGA, Proposition 2.5.1]). Let $V$ be a finite dimensional $\mathcal{U}_{q}\left(\mathfrak{p}_{J}\right)$-module. If

$$
\left[\mathrm{H}^{i}\left(\mathcal{U}_{q}\left(\mathfrak{u}_{J}\right), V\right): L^{q}(\lambda)\right] \neq 0
$$

then

$$
\left[\mathrm{H}^{i}\left(\mathcal{U}_{q}\left(\mathfrak{u}_{J}\right), \mathbb{C}\right) \otimes V: L^{q}(\lambda)\right] \neq 0 .
$$

Proof. Consider the case when $V$ is simple. Let $\mathcal{U}_{q}\left(\mathfrak{u}_{J}\right)_{+}$denote the augmentation ideal of $\mathcal{U}_{q}\left(\mathfrak{u}_{J}\right)$ as in [BNPP, 2.7$]$. Then $\mathcal{U}_{q}\left(\mathfrak{u}_{J}\right)_{+}$acts by zero on $V$. This is a consequence of two properties. First, $\mathcal{U}_{q}\left(\mathfrak{u}_{J}\right)$ is a normal subalgebra of $\mathcal{U}_{q}\left(\mathfrak{p}_{J}\right)$ (cf. BNPP, Corollary 2.7A]). Second, $\mathcal{U}_{q}\left(\mathfrak{u}_{J}\right)$ has only one irreducible representation, the trivial module. Taking $\mathcal{U}_{q}\left(\mathfrak{u}_{J}\right)_{+}$invariants, we have a non-zero (because of the second property) subspace of $V$ which is invariant under $\mathcal{U}_{q}\left(\mathfrak{p}_{J}\right)$ (by the first property). Thus $\mathcal{U}_{q}\left(\mathfrak{u}_{J}\right)_{+}$must act by zero on all of $V$, which implies that

$$
\mathrm{H}^{i}\left(\mathcal{U}_{q}\left(\mathfrak{u}_{J}\right), V\right) \cong \mathrm{H}^{i}\left(\mathcal{U}_{q}\left(\mathfrak{u}_{J}\right), \mathbb{C}\right) \otimes V
$$

Thus the statement holds in this case. The general argument follows by induction on the length of a composition series for $V$ and the long exact sequence in cohomology as in [UGA, Proposition 2.5.1].

\section{Euler characteristics}

4.1. Let $M$ be a module in $\mathcal{O}_{J}^{q}$. We denote the formal character of $M$ by ch $M$ (cf. [Jan1, 5A.8]). From [BNPP, Proposition 2.9.1(b)] and Theorem 3.5.2,

$$
\mathrm{H}^{n}\left(\mathcal{U}_{q}\left(\mathfrak{u}_{J}\right), M\right)=0
$$

for $n>\operatorname{dim} \mathfrak{u}_{J}$. Set

$$
\chi(M)=\sum_{n=0}^{\operatorname{dim}^{\mathfrak{u}_{J}}}(-1)^{n} \operatorname{ch~} \mathrm{H}^{n}\left(\mathcal{U}_{q}\left(\mathfrak{u}_{J}\right), M\right) .
$$

If $M$ has a trivial $\mathcal{U}_{q}\left(\mathfrak{u}_{J}\right)$-action, then

$$
\chi(M)=\sum_{n=0}^{\operatorname{dim} \mathfrak{u}_{J}}(-1)^{n} \operatorname{ch}\left(\mathrm{H}^{n}\left(\mathcal{U}_{q}\left(\mathfrak{u}_{J}\right), \mathbb{C}\right) \otimes M\right) .
$$


Now observe that if $0 \rightarrow M_{1} \rightarrow M \rightarrow M_{2} \rightarrow 0$ is a short exact sequence of $\mathcal{U}_{q}\left(\mathfrak{p}_{J}\right)$ modules where $M_{1}$ and $M_{2}$ have trivial $\mathcal{U}_{q}\left(\mathfrak{u}_{J}\right)$-action, then

$$
\begin{aligned}
\chi(M)= & \chi\left(M_{1}\right)+\chi\left(M_{2}\right) \\
= & \sum_{n=0}^{\operatorname{dim} \mathfrak{u}_{J}}(-1)^{n} \operatorname{ch}\left(\mathrm{H}^{n}\left(\mathcal{U}_{q}\left(\mathfrak{u}_{J}\right), \mathbb{C}\right) \otimes M_{1}\right) \\
& +\sum_{n=0}^{\operatorname{dim} \mathfrak{u}_{J}}(-1)^{n} \operatorname{ch}\left(\mathrm{H}^{n}\left(\mathcal{U}_{q}\left(\mathfrak{u}_{J}\right), \mathbb{C}\right) \otimes M_{2}\right) \\
= & \sum_{n=0}^{\operatorname{dim} \mathfrak{u}_{J}}(-1)^{n} \operatorname{ch}\left(\mathrm{H}^{n}\left(\mathcal{U}_{q}\left(\mathfrak{u}_{J}\right), \mathbb{C}\right) \otimes M\right) .
\end{aligned}
$$

By inducting on the composition length of $M$ and using the fact that all simple $\mathcal{U}_{q}\left(\mathfrak{p}_{J}\right)$-modules are obtained by inflating simple $\mathcal{U}_{q}\left(\mathfrak{l}_{J}\right)$-modules (by letting $\mathcal{U}_{q}\left(\mathfrak{u}_{J}\right)$ act trivially), the formula (4.1.1) holds for all $M$. In particular,

$$
\chi\left(L^{q}(\lambda)\right)=\sum_{n=0}^{\operatorname{dim} \mathfrak{u}_{J}}(-1)^{n} \operatorname{ch}\left(\mathrm{H}^{n}\left(\mathcal{U}_{q}\left(\mathfrak{u}_{J}\right), \mathbb{C}\right) \otimes L^{q}(\lambda)\right) .
$$

4.2. We recall the definition of the quantum exterior algebra $\Lambda_{q, J}^{\bullet}$ (cf. BNPP, $\S 2.9])$. Let $\Lambda_{q, J}^{\bullet}$ be the graded algebra with generators $\left\{x_{\alpha}: \alpha \in \Phi^{+} \backslash \Phi_{J}^{+}\right\}$, where $\operatorname{deg}\left(x_{\alpha}\right)=1$, satisfying the following relations:

$$
\begin{gathered}
x_{\alpha} \cdot x_{\beta}+q^{-\langle\alpha, \beta\rangle} x_{\beta} \cdot x_{\alpha}=0 \text { if } \alpha \prec \beta ; \\
x_{\alpha}^{2}=0 \text { for } \alpha \in \Phi^{+} \backslash \Phi_{J}^{+} .
\end{gathered}
$$

Here $\prec$ is the total ordering on the roots given by our fixed reduced expression of $w_{0, J}$ (cf. [BNPP, §2.4]).

We will now compare Euler characteristics in the quantum and classical cases. First observe that from (4.1.2) and BNPP, Proposition 2.9.1(a)],

$$
\begin{aligned}
\chi\left(L^{q}(\lambda)\right) & =\sum_{n=0}^{\operatorname{dim} \mathfrak{u}_{J}}(-1)^{n}\left[\operatorname{ch~} H^{n}\left(\mathcal{U}_{q}\left(\mathfrak{u}_{J}\right), \mathbb{C}\right)\right]\left[\operatorname{ch~} L^{q}(\lambda)\right] \\
& =\sum_{n=0}^{\operatorname{dim} \mathfrak{u}_{J}}(-1)^{n}\left[\operatorname{ch~} \Lambda_{q, J}^{n}\right]\left[\operatorname{ch~} L^{q}(\lambda)\right] .
\end{aligned}
$$

On the other hand, the analogous formula holds for the classical case:

$$
\chi(L(\lambda))=\sum_{n=0}^{\operatorname{dim} \mathfrak{u}_{J}}(-1)^{n}\left[\operatorname{ch~H}^{n}\left(\mathfrak{u}_{J}, \mathbb{C}\right)\right][\operatorname{ch} L(\lambda)]=\sum_{n=0}^{\operatorname{dim} \mathfrak{u}_{J}}(-1)^{n}\left[\operatorname{ch~} \Lambda^{n}\left(\mathfrak{u}_{J}^{*}\right)\right][\operatorname{ch} L(\lambda)] .
$$

It follows immediately from the definitions that for each $n$,

$$
\operatorname{ch} \Lambda_{q, J}^{n}=\operatorname{ch~} \Lambda^{n}\left(\mathfrak{u}_{J}^{*}\right) \text {. }
$$

Recalling (3.2.1), we have $\left[\operatorname{ch} \Lambda_{q, J}^{n}\right]\left[\operatorname{ch} L^{q}(\lambda)\right]=\left[\operatorname{ch~} \Lambda^{n}\left(\mathfrak{u}_{J}^{*}\right)\right][\operatorname{ch} L(\lambda)]$. Therefore,

$$
\sum_{n=0}^{\operatorname{dim} \mathfrak{u}_{J}}(-1)^{n} \operatorname{ch~} H^{n}\left(\mathcal{U}_{q}\left(\mathfrak{u}_{J}\right), L^{q}(\lambda)\right)=\sum_{n=0}^{\operatorname{dim} \mathfrak{u}_{J}}(-1)^{n} \operatorname{ch~} H^{n}\left(\mathfrak{u}_{J}, L(\lambda)\right) .
$$

Our strategy will be to use the fact that the right hand side of (4.2.3) is given by the classical Kostant's Theorem. 


\section{Kostant's THEOREM}

5.1. In this section we will prove an analog of Kostant's theorem for quantum groups. We begin by proving the result for trivial coefficients.

Theorem 5.1.1. Let $J \subseteq \Delta$ and $q$ be a generic parameter. Then as a $\mathcal{U}_{q}\left(\mathfrak{l}_{J}\right)$ module,

$$
\mathrm{H}^{n}\left(\mathcal{U}_{q}\left(\mathfrak{u}_{J}\right), \mathbb{C}\right) \cong \bigoplus_{\substack{w \in J \\ l(w)=n}} L_{J}^{q}(w \cdot 0) .
$$

Proof. According to Theorem 3.5.1, all $\mathcal{U}_{q}\left(\mathfrak{l}_{J}\right)$-composition factors of $\mathrm{H}^{n}\left(\mathcal{U}_{q}\left(\mathfrak{u}_{J}\right), \mathbb{C}\right)$ are of the form $L_{J}^{q}(w \cdot 0)$ where $w \in{ }^{J} W$. Furthermore, by BNPP, Proposition 2.9.1], if $w \cdot 0$ is a weight of $\mathrm{H}^{n}\left(\mathcal{U}_{q}\left(\mathfrak{u}_{J}\right), \mathbb{C}\right)$, then $w \cdot 0$ is a weight of $\Lambda_{q, J}^{n}$. Recall (e.g., from (4.2.2) and [UGA, Lemma 3.1.2]) that $\operatorname{dim}\left(\Lambda_{q, J}^{n}\right)_{w \cdot 0}=0$ for $l(w) \neq n$ and $\left(\Lambda_{q, J}^{n}\right)_{w \cdot 0} \cong k$ for $l(w)=n$.

Now by the classical Kostant's Theorem (cf. UGA, Theorem 4.2.1]), ch $\mathrm{L}_{q}(w \cdot 0)$ appears exactly once in $\sum_{n=0}^{\operatorname{dim} u_{J}}(-1)^{n} \operatorname{ch} \Lambda_{q, J}^{n}$; thus, it must appear exactly once in $\sum_{n=0}^{\operatorname{dim} \mathfrak{u}_{J}}(-1)^{n} \mathrm{ch} \mathrm{H}^{n}\left(\mathcal{U}_{q}\left(\mathfrak{u}_{J}\right), \mathbb{C}\right)$. But by the previous paragraph, $\left[\mathrm{H}^{n}\left(\mathcal{U}_{q}\left(\mathfrak{u}_{J}\right), \mathbb{C}\right)\right.$ : $\left.L_{J}^{q}(w \cdot 0)\right]=0$ for $l(w) \neq n$; therefore $\operatorname{ch~} L_{J}^{q}(w \cdot 0)$ must appear exactly once in ch $\mathrm{H}^{l(w)}\left(\mathcal{U}_{q}\left(\mathfrak{u}_{J}\right), \mathbb{C}\right)$ and $\left[\mathrm{H}^{l(w)}\left(\mathcal{U}_{q}\left(\mathfrak{u}_{J}\right), \mathbb{C}\right): L_{J}^{q}(w \cdot 0)\right]=1$. The result now follows because of the semisimplicity of the cohomology as a $\mathcal{U}_{q}\left(\mathfrak{l}_{J}\right)$-module.

5.2. We can now apply the preceding theorem to compute the cohomology of $\mathcal{U}_{q}\left(\mathfrak{u}_{J}\right)$ with coefficients in a finite dimensional simple $\mathcal{U}_{q}(\mathfrak{g})$-module.

Theorem 5.2.1. Let $J \subseteq \Delta, \mu \in X^{+}$and $q$ be a generic parameter. Then as a $\mathcal{U}_{q}\left(\mathfrak{l}_{J}\right)$-module,

$$
\mathrm{H}^{n}\left(\mathcal{U}_{q}\left(\mathfrak{u}_{J}\right), L^{q}(\mu)\right) \cong \bigoplus_{\substack{w \in J W \\ l(w)=n}} L_{J}^{q}(w \cdot \mu)
$$

Proof. First observe that by Theorem 3.5.1, all $\mathcal{U}_{q}\left(\mathfrak{l}_{J}\right)$-composition factors of $\mathrm{H}^{n}\left(\mathcal{U}_{q}\left(\mathfrak{u}_{J}\right), L^{q}(\mu)\right)$ are of the form $L_{J}^{q}(w \cdot \mu)$ where $w \in{ }^{J} W$. Next observe that if $\left[\mathrm{H}^{n}\left(\mathcal{U}_{q}\left(\mathfrak{u}_{J}\right), L^{q}(\mu)\right): L_{J}^{q}(w \cdot \mu)\right] \neq 0$, then $\left[\mathrm{H}^{n}\left(\mathcal{U}_{q}\left(\mathfrak{u}_{J}\right), \mathbb{C}\right) \otimes L^{q}(\mu): L_{J}^{q}(w \cdot \mu)\right] \neq 0$. In other words,

$$
\operatorname{Hom}_{U_{q}\left(\mathfrak{l}_{J}\right)}\left(L_{J}^{q}(w \cdot \mu), \mathrm{H}^{n}\left(\mathcal{U}_{q}\left(\mathfrak{u}_{J}\right), \mathbb{C}\right) \otimes L^{q}(\mu)\right) \neq 0 .
$$

We are now in a position to use the argument in [UGA, Theorem 4.2.1]. We first apply Frobenius reciprocity and Theorem 5.1.1 to deduce that

$$
w \cdot \mu=w^{\prime} \cdot 0+\nu
$$

where $\nu \in \operatorname{wt}\left(L^{q}(\mu)\right)$ and $l\left(w^{\prime}\right)=n$. The argument in [UGA shows that $w=w^{\prime}$, thus $l(w)=n$. Furthermore,

$$
\mathrm{H}^{n}\left(\mathcal{U}_{q}\left(\mathfrak{u}_{J}\right), \mathbb{C}\right)_{w \cdot 0}=1
$$

We can now conclude that

$$
\left[\mathrm{H}^{n}\left(\mathcal{U}_{q}\left(\mathfrak{u}_{J}\right), L^{q}(\mu)\right): L_{J}^{q}(w \cdot \mu)\right] \leq 1
$$

for all $w \in{ }^{J} W$. 
The cohomology is completely reducible as a $\mathcal{U}_{q}\left(\mathfrak{l}_{J}\right)$-module, so it remains to prove that

$$
\left[\mathrm{H}^{n}\left(\mathcal{U}_{q}\left(\mathfrak{u}_{J}\right), L^{q}(\mu)\right): L_{J}^{q}(w \cdot \mu)\right]=1
$$

for all $w \in{ }^{J} W$ and $l(w)=n$. Suppose that $w \in{ }^{J} W$ and $l(w)=n$. By Kostant's Theorem in the classical case, $\operatorname{ch} L(w \cdot \mu)$ occurs exactly once in the $\operatorname{sum} \sum_{i=0}^{\operatorname{dim} \mathfrak{u}_{J}}(-1)^{i} \operatorname{ch~} \mathrm{H}^{i}\left(\mathfrak{u}_{J}, L(\mu)\right)$. From (4.2.3) , ch $L_{J}^{q}(w \cdot \mu)$ appears exactly once in $\sum_{i=0}^{\operatorname{dim} \mathfrak{u}_{J}}(-1)^{i} \operatorname{ch~} \mathrm{H}^{i}\left(\mathcal{U}_{q}\left(\mathfrak{u}_{J}\right), L^{q}(\lambda)\right)$. From the preceding paragraph $L_{J}^{q}(w$. $\mu)$ can only appear as a composition factor, with multiplicity at most one, in $\mathrm{H}^{i}\left(\mathcal{U}_{q}\left(\mathfrak{u}_{J}\right), L^{q}(\lambda)\right)$ where $i=n=l(w)$. Putting these statements together implies that $\left[\mathrm{H}^{n}\left(\mathcal{U}_{q}\left(\mathfrak{u}_{J}\right), L^{q}(\mu)\right): L_{J}^{q}(w \cdot \mu)\right]=1$.

\section{RoOTS OF UNITY}

6.1. In this section we will demonstrate that Kostant's theorem has a quantum analog in the case when the parameter is a primitive $l$ th root of unity under the conditions that $l \geq h-1$ and that the highest weight of the simple $\mathcal{U}_{\zeta}(\mathfrak{g})$-module is in the bottom alcove. These results were inspired by Polo and Tilouine's result for reductive groups in positive characteristic (cf. [PT, Theorem 2.1], [UGA, Theorem 4.2.1]).

6.2. The first step is to prove an upper bound on the composition factors of the cohomology groups $\mathrm{H}^{\bullet}\left(\mathcal{U}_{\zeta}\left(\mathfrak{u}_{J}\right), L^{\zeta}(\mu)\right)$. In order to do so we need some additional notation. Since $u_{\zeta}(\mathfrak{g})$ is a normal sub Hopf algebra of $U_{\zeta}(\mathfrak{g})$, one can form the algebra $K u_{\zeta}(\mathfrak{g})$ where $K$ is the subalgebra of $U_{\zeta}(\mathfrak{g})$ generated by $\left\{K_{\alpha}^{ \pm 1}: \alpha \in \Delta\right\}$. The same construction works if one replaces $\mathfrak{g}$ by $\mathfrak{b}, \mathfrak{p}_{J}$ or $\mathfrak{l}_{J}$. This construction is dual to the construction given in [PW] Chapter 9] and analogous to the graded $G_{1} T$-category for reductive algebraic group schemes $G$ in positive characteristic. For a fixed $l>1$, let $W_{l}=W \ltimes l \mathbb{Z} \Phi$, a subgroup of the affine Weyl group, and $\widehat{W}_{l}=W \ltimes l X$, a subgroup of the extended affine Weyl group. Write $X_{\mathrm{res}}^{+}$for the set of $l$-restricted weights

$$
\{\lambda \in X: 0 \leq\langle\lambda, \check{\alpha}\rangle<l \text { for all } \alpha \in \Delta\}
$$

and similarly $X_{J, \text { res }}^{+}$for the set of $l$-restricted $J$-weights. Let $\bar{C}_{\mathbb{Z}}$ be the (closed) bottom $l$-alcove.

The following theorem provides information about the $\mathcal{U}_{\zeta}\left(\mathfrak{l}_{J}\right)$-composition factors in the $\mathcal{U}_{\zeta}\left(\mathfrak{u}_{J}\right)$-cohomology.

Theorem 6.2.1. Let $J \subseteq \Delta$ and $\zeta$ be a primitive lth root of unity.

(a) If $\left[\mathrm{H}^{i}\left(\mathcal{U}_{\zeta}\left(\mathfrak{u}_{J}\right), L^{\zeta}(\mu)\right): L_{J}^{\zeta}(\sigma)\right]_{\mathcal{U}_{\zeta}\left(\mathfrak{I}_{J}\right)} \neq 0$ where $\mu \in X^{+}$, then $\mu=w \cdot \sigma$ where $w \in \widehat{W}_{l}$.

(b) If $\left[\mathrm{H}^{i}\left(\mathcal{U}_{\zeta}\left(\mathfrak{u}_{J}\right), L^{\zeta}(\mu)\right): L_{J}^{\zeta}(\sigma)\right] \mathcal{U}_{\zeta}\left(l_{J}\right) \neq 0$ where $\mu \in X_{\text {res }}^{+}$and $\sigma \in X_{J, \text { res }}^{+}$, then $\mu=w \cdot \sigma$ where $w \in W_{l}$.

Proof. We will provide a sketch of the argument, which is similar to the one given in [UGA, Theorem 2.4.1]. It should be noted that one needs to deviate somewhat from the original proof for Frobenius kernels because a different spectral sequence construction is needed in the quantum case.

(a) The first part of the proof follows by using the argument provided in UGA, Theorem 2.4.1], replacing $L_{J}$ with $\mathcal{U}_{\zeta}\left(\mathfrak{l}_{J}\right)$ and $\left(L_{J}\right)_{1} T$ with the algebra $K u_{\zeta}\left(\mathfrak{l}_{J}\right)$, etc. 
The main points are that if $\left.\left[\mathrm{H}^{i}\left(\mathcal{U}_{\zeta}\left(\mathfrak{u}_{J}\right), L^{\zeta}(\mu)\right): L_{J}^{\zeta}(\sigma)\right]\right]_{\mathcal{U}_{\zeta}\left(\mathfrak{l}_{J}\right)} \neq 0$ where $\mu \in X^{+}$, then one can write $\mu=\mu_{0}+l \mu_{1}$ where $\mu_{0} \in X_{\text {res }}^{+}, \mu_{1} \in X^{+}$, and $\sigma=\sigma_{0}+l \sigma_{1}$ where $\sigma_{0} \in X_{J, \mathrm{res}}^{+}, \sigma_{1} \in X_{J}^{+}$. Then one shows that

$$
\left[\mathrm{H}^{i}\left(\mathcal{U}_{\zeta}\left(\mathfrak{u}_{J}\right), L^{\zeta}\left(\mu_{0}\right)\right): L_{J}^{\zeta}\left(\sigma_{0}\right) \otimes l \gamma\right]_{K u_{\zeta}\left(\mathfrak{l}_{J}\right)} \neq 0
$$

for some $\gamma \in X$. Here $\gamma$ is the difference between a weight in the simple $\mathcal{U}_{\zeta}(\mathfrak{g})$ module $L^{\zeta}\left(\mu_{1}\right)$ and a weight in the simple $\mathcal{U}_{\zeta}\left(\mathfrak{l}_{J}\right)$-module $L_{J}^{\zeta}\left(\sigma_{1}\right)$.

Here is where the proof now departs from the one given in UGA, Theorem 2.4.1(a)]. Let $\mathcal{Z}$ be the subalgebra in $\mathcal{U}_{\zeta}(\mathfrak{g})$ generated by $\left\{E_{\gamma}^{l}, F_{\gamma}^{l}: \gamma \in \Phi\right\}$ and set $\mathcal{Z}_{J}=\mathcal{U}_{\zeta}\left(\mathfrak{u}_{J}\right) \cap \mathcal{Z}$. Then $\mathcal{U}_{\zeta}\left(\mathfrak{u}_{J}\right) / / \mathcal{Z}_{J} \cong u_{\zeta}\left(\mathfrak{u}_{J}\right)$. From [BNPP, Section 5.4] there exists a spectral sequence

$$
E_{2}^{a, b}=\mathrm{H}^{a}\left(u_{\zeta}\left(\mathfrak{u}_{J}\right), \mathrm{H}^{b}\left(\mathcal{Z}_{J}, L^{\zeta}\left(\mu_{0}\right)\right)\right) \Rightarrow \mathrm{H}^{a+b}\left(\mathcal{U}_{\zeta}\left(\mathfrak{u}_{J}\right), L^{\zeta}\left(\mu_{0}\right)\right) .
$$

Since the algebra $\mathcal{Z}_{J}$ is central and generated by nilpotent elements, the action of $\mathcal{Z}_{J}$ on $L^{\zeta}(\mu)$ is trivial. Moreover, the action of $u_{\zeta}\left(\mathfrak{u}_{J}\right)$ on $\mathrm{H}^{\bullet}\left(\mathcal{Z}_{J}, \mathbb{C}\right)$ is trivial. Therefore, one can rewrite the spectral sequence as

$$
E_{2}^{a, b}=\mathrm{H}^{a}\left(u_{\zeta}\left(\mathfrak{u}_{J}\right), L^{\zeta}\left(\mu_{0}\right)\right) \otimes \mathrm{H}^{b}\left(\mathcal{Z}_{J}, \mathbb{C}\right) \Rightarrow \mathrm{H}^{a+b}\left(\mathcal{U}_{\zeta}\left(\mathfrak{u}_{J}\right), L^{\zeta}\left(\mu_{0}\right)\right) .
$$

Now suppose that $\left[\mathrm{H}^{i}\left(\mathcal{U}_{\zeta}\left(\mathfrak{u}_{J}\right), L^{\zeta}\left(\mu_{0}\right)\right): L_{J}^{\zeta}\left(\sigma_{0}\right) \otimes l \gamma\right]_{K u_{\zeta}\left(\mathfrak{l}_{J}\right)} \neq 0$. Then

$$
\operatorname{Hom}_{K u_{\zeta}\left(\mathfrak{l}_{J}\right)}\left(P, \mathrm{H}^{i}\left(\mathcal{U}_{\zeta}\left(\mathfrak{u}_{J}\right), L^{\zeta}\left(\mu_{0}\right)\right)\right) \neq 0
$$

where $P:=P_{J}^{\zeta}\left(\sigma_{0}\right) \otimes l \gamma$ is the $K u_{\zeta}\left(\mathfrak{l}_{J}\right)$-projective cover of $L_{J}^{\zeta}\left(\sigma_{0}\right) \otimes l \gamma$. From the spectral sequence one can deduce that

$$
\operatorname{Hom}_{K u_{\zeta}\left(\mathfrak{l}_{J}\right)}\left(P, \mathrm{H}^{a}\left(u_{\zeta}\left(\mathfrak{u}_{J}\right), L^{\zeta}\left(\mu_{0}\right)\right) \otimes \mathrm{H}^{b}\left(\mathcal{Z}_{J}, \mathbb{C}\right)\right) \neq 0
$$

where $i=a+b$.

The weights of $\mathrm{H}^{\bullet}\left(\mathcal{Z}_{J}, \mathbb{C}\right)$ are of the form $l \delta$ where $\delta \in \mathbb{Z} \Phi$. This implies that

$$
\begin{aligned}
0 & \neq \operatorname{dim}_{\operatorname{Hom}} \operatorname{Hu}_{\zeta}\left(\mathfrak{l}_{J}\right) \\
& =\left[P, \mathrm{H}^{a}\left(u_{\zeta}\left(u_{\zeta}\left(\mathfrak{u}_{J}\right), L^{\zeta}\left(\mu_{0}\right)\right) \otimes l \delta\right)\right. \\
& \left.\left.=\operatorname{dim}^{\zeta}\left(\mu_{0}\right)\right): L_{J}^{\zeta}\left(\sigma_{0}\right) \otimes l \gamma \otimes(-l \delta)\right]_{K u_{\zeta}(\mathfrak{g})}\left(\operatorname{coind}_{K u_{\zeta}\left(\mathfrak{l}_{J}\right)}^{K u_{\zeta}(\mathfrak{g})} P \otimes(-l \delta), L^{\zeta}\left(\mu_{0}\right)\right),
\end{aligned}
$$

where the last equality is deduced via an argument using the Lyndon-HochschildSerre spectral sequence.

Therefore, the linkage principle for blocks in $K u_{\zeta}(\mathfrak{g})$ implies that $\sigma_{0}+l \gamma-l \delta$ and $\mu_{0}$ are linked under $W_{l}$; thus $\sigma_{0}$ and $\mu_{0}$ are linked under $\widehat{W}_{l}$.

(b) Under the hypotheses, we have $\gamma=0$ as in the proof of [UGA, Theorem 2.4.1]. In this case $\mu=\mu_{0}$ and $\sigma=\sigma_{0}$, and since $\delta \in \mathbb{Z} \Phi$, we deduce that $\mu=w \cdot \sigma$ where $w \in W_{l}$.

6.3. One can verify that the proofs of UGA, Proposition 3.5.1, Theorem 3.6.1] depend only on using weight estimates which apply to our setting. A conversion of these results to the quantum setting yields the following results.

Proposition 6.3.1. Let $\zeta$ be a primitive lth root of unity with $l \geq h-1$.

(a) Suppose $\sigma=w \cdot 0+l \mu$ is a weight of $\Lambda_{\zeta, J}^{\bullet}$ where $w \in W$ and $\mu \in X$. Then $\sigma=x \cdot 0$ for some $x \in W$.

(b) If $w \in{ }^{J} W$, then $L_{J}^{\zeta}(w \cdot 0)$ is in the bottom alcove for $\mathcal{U}_{\zeta}\left(\mathfrak{l}_{J}\right)$. 
(c) If $w \in{ }^{J} W$ and $\lambda \in \bar{C}_{\mathbb{Z}} \cap X^{+}$, then $L_{J}^{\zeta}(w \cdot 0) \otimes L^{\zeta}(\lambda)$ is completely reducible as a $\mathcal{U}_{\zeta}\left(\mathfrak{l}_{J}\right)$-module.

We also need to revisit Section 4 on Euler characteristics. The formula (4.2.1) and $\mathrm{BNPP}$, Lemma 2.9.1(a)] hold when $q$ is a root of unity. Therefore,

$$
\chi\left(L^{\zeta}(\lambda)\right)=\sum_{n=0}^{\operatorname{dim} \mathfrak{u}_{J}}(-1)^{n}\left[\operatorname{ch~} \Lambda_{\zeta, J}^{n}\right]\left[\operatorname{ch~} L^{\zeta}(\lambda)\right] .
$$

Observe that if $\lambda \in \bar{C}_{\mathbb{Z}} \cap X^{+}$, then for each $n$,

$$
\left[\operatorname{ch~} \Lambda_{q, J}^{n}\right]\left[\operatorname{ch~} L_{\zeta}(\lambda)\right]=\left[\operatorname{ch~} \Lambda^{n}\left(\mathfrak{u}_{J}^{*}\right)\right][\operatorname{ch} L(\lambda)] .
$$

So it follows from the argument in Section 4.2 that for $\mu \in \bar{C}_{\mathbb{Z}} \cap X^{+}$,

$$
\sum_{n=0}^{\operatorname{dim}_{\mathfrak{u}_{J}}}(-1)^{n} \operatorname{ch~} \mathrm{H}^{n}\left(\mathcal{U}_{\zeta}\left(\mathfrak{u}_{J}\right), L^{\zeta}(\mu)\right)=\sum_{n=0}^{\operatorname{dim} \mathfrak{u}_{J}}(-1)^{n} \operatorname{ch~} H^{n}\left(\mathfrak{u}_{J}, L(\mu)\right) .
$$

6.4. We are now in a position to directly apply the proofs of Theorems 5.1.1 and 5.2 .1 by setting $q=\zeta$, with $\zeta$ being a primitive $l$ th root of unity where $l \geq h-1$, to prove a version of Kostant's Theorem in the root of unity case.

Theorem 6.4.1. Let $J \subseteq \Delta, \mu \in X^{+}$and $\zeta$ be a primitive lth root of unity. Assume that $l \geq h-1$ and $\mu \in \bar{C}_{\mathbb{Z}}$. Then as a $\mathcal{U}_{\zeta}\left(\mathfrak{l}_{J}\right)$-module,

$$
\mathrm{H}^{n}\left(\mathcal{U}_{\zeta}\left(\mathfrak{u}_{J}\right), L^{\zeta}(\mu)\right) \cong \bigoplus_{\substack{w \in J W \\ l(w)=n}} L_{J}^{\zeta}(w \cdot \mu)
$$

6.5. We remark that one can also prove a partial converse of Kostant's theorem as demonstrated in [UGA, Theorem 5.1.1].

Theorem 6.5.1. Let $\zeta$ be a primitive lth root of unity with $l<h-1$ and $q$ a generic parameter. Then

$$
\operatorname{ch~}^{\bullet}\left(\mathcal{U}_{\zeta}(\mathfrak{u}), \mathbb{C}\right) \neq \operatorname{ch~} \mathrm{H}^{\bullet}\left(\mathcal{U}_{q}(\mathfrak{u}), \mathbb{C}\right) .
$$

As is the case for comparing cohomology for complex semisimple Lie algebras versus their modular analogs, the comparison between the cohomology for arbitrary unipotent radicals of parabolic subalgebras and their quantum analogs is much more subtle when $l<h-1$ (cf. [UGA, §6]).

\section{VigRe Algebra Group at the University of Georgia}

This project was initiated during Fall Semester 2007 under the Vertical Integration of Research and Education (VIGRE) Program sponsored by the National Science Foundation (NSF) at the Department of Mathematics at the University of Georgia (UGA). We would like to acknowledge the NSF VIGRE grants DMS0089927 and DMS-0738586 for partial financial support of this project. The VIGRE Algebra Group consists of 3 faculty members, 1 postdoctoral fellow, and 6 graduate students. The group is led by Brian D. Boe, Leonard Chastkofsky and Daniel K. Nakano. The email addresses of the members of the group are given below. 
Faculty:

Brian D. Boe

Leonard Chastkofsky

Daniel K. Nakano

Postdoctoral Fellow:

Benjamin Jones

Graduate Students:

Irfan Bagci

Benjamin Connell

Wenjing Li

Kenyon J. Platt

Jae-Ho Shin

Caroline B. Wright brian@math.uga.edu

lenny@math.uga.edu

nakano@math.uga.edu

bjones@math.uga.edu

bagci@math.uga.edu

bconnell@math.uga.edu

wli@math.uga.edu

platt@math.uga.edu

jshin@math.uga.edu

cwright@math.uga.edu

\section{REFERENCES}

[BNPP] Christopher P. Bendel, Daniel K. Nakano, Brian J. Parshall, and Cornelius Pillen, Cohomology for quantum groups via the geometry of the nullcone, preprint, 2007.

$[\mathrm{BNW}]$ Brian D. Boe, Daniel K. Nakano, and Emilie Wiesner, Category $\mathcal{O}$ for the Virasoro algebra: cohomology and Koszulity, Pacific J. Math. 234 (2008), no. 1, 1-21. MR2375311

[DCK] Corrado De Concini and Victor G. Kac, Representations of quantum groups at roots of 1, Operator algebras, unitary representations, enveloping algebras, and invariant theory (Paris, 1989), Progress in Mathematics, vol. 92, Birkhäuser, Boston, MA, 1990, pp. 471506. MR 1103601 (92g:17012)

[FP] Eric M. Friedlander and Brian J. Parshall, Cohomology of infinitesimal and discrete groups, Math. Ann. 273 (1986), no. 3, 353-374. MR824427 (87e:22026)

[GK] Victor Ginzburg and Shrawan Kumar, Cohomology of quantum groups at roots of unity, Duke Math. J. 69 (1993), no. 1, 179-198. MR1201697 (94c:17026)

[GW] Roe Goodman and Nolan R. Wallach, Representations and invariants of the classical groups, Encyclopedia of Mathematics and its Applications, vol. 68, Cambridge University Press, Cambridge, 1998. MR1606831 (99b:20073)

[HeK] István Heckenberger and Stefan Kolb, On the Bernstein-Gelfand-Gelfand resolution for Kac-Moody algebras and quantized enveloping algebras, Transform. Groups 12 (2007), no. 4, 647-655. MR2365438(2008k:17033)

[Hoc] Gerald Hochschild, Relative homological algebra, Trans. Amer. Math. Soc. 82 (1956), 246-269. MR0080654(18:278a)

[HK] Jin Hong and Seok-Jin Kang, Introduction to quantum groups and crystal bases, Graduate Studies in Mathematics, vol. 42, American Mathematical Society, Providence, RI, 2002. MR 1881971(2002m:17012)

[Hum] James E. Humphreys, Representations of semisimple Lie algebras in the BGG category $\mathcal{O}$, Graduate Studies in Mathematics, vol. 94, American Mathematical Society, Providence, RI, 2008. MR2428237 (2009f:17013)

[Jan1] Jens Carsten Jantzen, Lectures on quantum groups, Graduate Studies in Mathematics, vol. 6, American Mathematical Society, Providence, RI, 1996. MR1359532 (96m:17029)

[Jan2] - Representations of algebraic groups, second ed., Mathematical Surveys and Monographs, vol. 107, American Mathematical Society, Providence, RI, 2003. MR2015057 (2004h:20061)

[Jan3] Nilpotent orbits in representation theory, Lie theory, Progress in Mathematics, vol. 228, Birkhäuser, Boston, MA, 2004, pp. 1-211. MR2042689(2005c:14055)

[Kna] Anthony W. Knapp, Lie groups, Lie algebras, and cohomology, Mathematical Notes, vol. 34, Princeton University Press, Princeton, NJ, 1988. MR 938524 (89j:22034) 
[Kum] Shrawan Kumar, Kac-Moody groups, their flag varieties and representation theory, Progress in Mathematics, vol. 204, Birkhäuser, Boston, MA, 2002. MR.1923198 (2003k:22022)

[Mal] Fyodor Malikov, Quantum groups: singular vectors and BGG resolution, Infinite analysis, Parts A and B (Kyoto, 1991), Adv. Ser. Math. Phys., vol. 16, World Scientific, River Edge, NJ, 1992, pp. 623-643. MR1187567 (93k:17034)

[PW] Brian Parshall and Jian Pan Wang, Quantum linear groups, Mem. Amer. Math. Soc. 89 (1991), no. 439. MR1048073 (91g:16028)

[PT] Patrick Polo and Jacques Tilouine, Bernstein-Gelfand-Gelfand complexes and cohomology of nilpotent groups over $\mathbb{Z}_{(p)}$ for representations with p-small weights, Astérisque (2002), no. 280, Cohomology of Siegel varieties, 97-135. MR1944175 (2003j:17027)

[RC] Alvany Rocha-Caridi, Splitting criteria for $\mathfrak{g}$-modules induced from a parabolic and the Berñstein-Gel'fand-Gel'fand resolution of a finite-dimensional, irreducible $\mathfrak{g}$-module, Trans. Amer. Math. Soc. 262 (1980), no. 2, 335-366. MR.586721 (82f:17006)

[UGA] University of Georgia VIGRE Algebra Group, On Kostant's theorem for Lie algebra cohomology, Cont. Math. (2009), no. 478, 39-60.

Department of Mathematics, University of Georgia, Athens, Georgia 30602 